\title{
Bottom (70,1-) baryon multiplet
}

\author{
Elena Matskevich ${ }^{1, *}$ \\ ${ }^{1}$ St. Petersburg Mining University, Maliy V.O. Pr., 83, St. Petersburg, 199226, Russia
}

\begin{abstract}
The aim of this paper is to derive systems of equations for the amplitudes for the case of negative parity (70,1-) bottom nonstrange baryons and to calculate the masses of these particles. In order to calculate masses of $(70,1-)$ bottom baryons we use the relativistic quark model. The relativistic three-quark equations of the (70,1-) bottom baryon multiplet are derived in the framework of the dispersion relation technique. The relativistic three-quark equations of the (70,1-) bottom baryon multiplet are derived. The approximate solutions of these equations using the method based on the extraction of leading singularities of the amplitude are obtained. The masses of 21 baryons are predicted.
\end{abstract}

\section{Introduction}

Hadron spectroscopy has always played an important role in the revealing mechanisms underlying the dynamic of strong interactions. At low energies, typical for baryon spectroscopy, QCD does not admit a perturbative expansion in the strong coupling constant. In 1974 't Hooft [1] suggested a perturbative expansion of QCD in terms of the parameter $1 / N c$ where $N c$ is the number of colors. This suggestion together with the power counting rules of Witten [2] has lead to the $1 / N c$ expansion method which allows to systematically analyse baryon properties.

In the series of papers $[3,4,5,6,7]$ a practical treatment of relativistic three-hadron systems have been developed. The physics of three-hadron system is usefully described in term of the pairwise interactions among the three particles. The theory is based on the two principles of unitarity and analyticity, as applied to the two-body subenergy channels. The linear integral equations in a single variable are obtained for the isobar amplitudes. The coupled integral equations are solved in terms of simple algebra.

In our paper [8] relativistic generalization of the three-body Faddeev equations was obtained in the form of dispersion relations in the pair energy of two interacting particles. The mass spectrum of S-wave baryons including $u, d, s$-quarks was calculated by a method based on isolating the leading singularities in the amplitude. We searched for the approximate solution of integral three-quark equations by taking into account two-particle and triangle singularities, all the weaker ones being neglected. If we considered such an approximation, which corresponds to taking into account two-body and triangle singularities, and defined all the smooth functions of the middle point of the physical region

\footnotetext{
*Corresponding author : ematskev@mail.ru
} 
of Dalitz-plot, then the problem was reduced to the one of solving a system of simple algebraic equations.

In our paper [9] the construction of the orbital-flavor-spin wave functions for the (70,1-) multiplet are given. We deal with a three-quark system having one unit of orbital excitation. The orbital part of wave function must have a mixed symmetry. The spin-flavor part of wave function must have the same symmetry is order to obtain a totally symmetric state in the orbital-flavor-spin space. The integral equations using the orbital-flavor-spin wave functions was constructed. It allows to calculate the mass spectra for all baryons of (70,1-) multiplet. The 15 resonances are in good agreement with experimental data. We have predicted 15 masses of baryons. In our model the four parameter are used: gluon coupling constants $g_{+}$and $g_{-}$for the various parity, cutoff energy parameters $\lambda, \lambda_{s}$ for the nonstrange and strange diquarks.

In the paper [10] the relativistic three-quark equations of the excited $(70,1-)$ charmed baryons are found in the framework of the dispersion relation technique. The $(70,1-)$ charmed baryon multiplet has 23 baryons with different masses. The 6 resonances are in good agreement with the experimental data. We have predicted 17 masses of charmed excited baryons.

In the framework of the proposed approximate method of solving the relativistic threeparticle problem, we have obtained a spectrum of $P$-wave bottom baryons. This paper is a generalization of our works $[9,10]$.

The paper is organized as follows. In Sect. II the relativistic three-quark equations are obtained in the form of the dispersion relation over the two-body subenergy.

In Sect. III the systems of equations for the reduced amplitudes are derived.

Section IV is devoted to the calculation results for the mass spectrum of the (70,1-) bottom multiplet (Tables I-IV).

\section{The three-quark integral equations for the (70,1-) bottom multiplet}

We obtain the system integral equations for the $\Sigma_{b}$ state with the $J^{P}=\frac{3}{2}^{-}(10,2)$ multiplet:

$$
\begin{gathered}
A_{1}^{0}\left(s, s_{12}\right)=\lambda b_{1^{+}}\left(s_{12}\right) L_{1^{+}}\left(s_{12}\right)+K_{1^{+}}\left(s_{12}\right)\left[\frac{1}{4} A_{1}^{0 b}\left(s, s_{13}\right)+\frac{3}{4} A_{0}^{1 b}\left(s, s_{13}\right)\right]+ \\
+K_{1^{+}}\left(s_{12}\right)\left[\frac{1}{4} A_{1}^{0 b}\left(s, s_{23}\right)+\frac{3}{4} A_{0}^{1 b}\left(s, s_{23}\right)\right] \\
A_{1}^{0 b}\left(s, s_{13}\right)=\lambda b_{1_{b}^{+}}\left(s_{13}\right) L_{1_{b}^{+}}\left(s_{13}\right)+K_{1_{b}^{+}}\left(s_{13}\right)\left[\frac{1}{2} A_{1}^{0}\left(s, s_{12}\right)-\frac{1}{4} A_{1}^{0 b}\left(s, s_{12}\right)\right]+ \\
+K_{1_{b}^{+}}\left(s_{13}\right)\left[\frac{3}{4} A_{0}^{1 b}\left(s, s_{23}\right)+\frac{1}{2} A_{1}^{0}\left(s, s_{23}\right)-\frac{1}{4} A_{1}^{0 b}\left(s, s_{23}\right)+\frac{3}{4} A_{0}^{1 b}\left(s, s_{23}\right)\right]
\end{gathered}
$$




$$
\begin{aligned}
A_{0}^{1 b}\left(s, s_{23}\right) & =\lambda b_{1_{b}^{-}}\left(s_{23}\right) L_{1_{b}^{-}}\left(s_{23}\right)+K_{1_{b}^{-}}\left(s_{23}\right)\left[\frac{1}{2} A_{1}^{0}\left(s, s_{12}\right)+\frac{1}{4} A_{1}^{0 b}\left(s, s_{12}\right)\right]+ \\
+ & K_{1_{b}^{-}}\left(s_{23}\right)\left[\frac{1}{4} A_{0}^{1 b}\left(s, s_{12}\right)+\frac{1}{2} A_{1}^{0}\left(s, s_{13}\right)+\frac{1}{4} A_{1}^{0 b}\left(s, s_{13}\right)+\frac{1}{4} A_{0}^{1 b}\left(s, s_{13}\right)\right]
\end{aligned}
$$

Here function $L_{J}\left(s_{i k}\right)$ has the form

$$
L_{J}\left(s_{i k}\right)=\frac{G_{J}\left(s_{i k}\right)}{1-B_{J}\left(s_{i k}\right)}
$$

The integral operator $K_{J}\left(s_{i k}\right)$ is:

$$
K_{J}\left(s_{i k}\right)=L_{J}\left(s_{i k}\right) \int_{\left(m_{i}+m_{k}\right)^{2}}^{\Lambda_{J} \frac{\left(m_{i}+m_{k}\right)^{2}}{\pi}} \frac{d s_{i k}^{\prime}}{\pi} \frac{\rho_{J}\left(s_{i k}^{\prime}\right) G_{J}\left(s_{i k}^{\prime}\right)}{s^{\prime}{ }_{i k}-s_{i k}} \int_{-1}^{1} \frac{d z}{2}
$$

We can replace integral equations by the algebraic equations for the state $\Sigma_{b}$ $J^{P}=\frac{3}{2}^{-}$of the $(10,2)$ multiplet:

$$
\begin{gathered}
\alpha_{1}^{0}\left(s, s_{0}\right)=\lambda+\frac{1}{2} \alpha_{1}^{0 b}\left(s, s_{0}\right) I_{1^{+} 1_{b}^{+}}\left(s, s_{0}\right) \frac{b_{1_{b}^{+}}\left(s_{0}\right)}{b_{1^{+}}\left(s_{0}\right)}+\frac{3}{2} \alpha_{0}^{1 b}\left(s, s_{0}\right) I_{1^{+} 1_{b}^{-}}\left(s, s_{0}\right) \frac{b_{1_{b}^{-}}\left(s_{0}\right)}{b_{1^{+}}\left(s_{0}\right)} \\
\alpha_{1}^{0 b}\left(s, s_{0}\right)=\lambda+\alpha_{1}^{0}\left(s, s_{0}\right) I_{1_{b}^{+} 1^{+}}\left(s, s_{0}\right) \frac{b_{1^{+}}\left(s_{0}\right)}{b_{1_{b}^{+}}\left(s_{0}\right)}-\frac{1}{2} \alpha_{1}^{0 b}\left(s, s_{0}\right) I_{1_{b}^{+} 1_{b}^{+}}\left(s, s_{0}\right) \\
+\frac{3}{2} \alpha_{0}^{1 b}\left(s, s_{0}\right) I_{1_{b}^{+} 1_{b}^{-}}\left(s, s_{0}\right) \frac{b_{1_{b}^{-}}\left(s_{0}\right)}{b_{1_{b}^{+}}\left(s_{0}\right)} \\
\alpha_{0}^{1 b}\left(s, s_{0}\right)=\lambda+\alpha_{1}^{0}\left(s, s_{0}\right) I_{1_{b}^{-1^{+}}}\left(s, s_{0}\right) \frac{b_{1^{+}}\left(s_{0}\right)}{b_{1_{b}^{-}}\left(s_{0}\right)}+\frac{1}{2} \alpha_{1}^{0 b}\left(s, s_{0}\right) I_{1_{b}^{-} 1_{b}^{+}}\left(s, s_{0}\right) \frac{b_{1_{b}^{+}}\left(s_{0}\right)}{b_{1_{b}^{-}}\left(s_{0}\right)} \\
+\frac{1}{2} \alpha_{0}^{1 b}\left(s, s_{0}\right) I_{1_{b}^{-1} 1_{b}^{-}}\left(s, s_{0}\right)
\end{gathered}
$$

Here the reduced amplitudes for the diquarks $1^{+}, 1_{b}^{+}, 1_{b}^{-}$are given. The function $I_{J_{1} J_{2}}\left(s, s_{0}\right)$ takes into account singularity which corresponds to the simultaneous vanishing of all propagators in the triangle diagrams. 
We calculate the system equations and can determine the mass values of the $\Sigma_{b}$ $J^{P}=\frac{3}{2}^{-}(10,2)$. We calculate a pole in $s$ which corresponds to the bound state of three quarks.

By analogy with $\Sigma_{b}$-hyperon we obtain the system equations for the reduced amplitudes for all particles (70,1-) multiplets.

\section{Calculation results}

The present paper is an extension of previous research works devoted to strange and charmed $P$-wave $(70,1-)$ baryon multiplets. Therefore we take the part of parameters from those ones. The mass of $u, d$ quarks is equal to $m_{u_{i} d}=570 \mathrm{MeV}$ as in those works, the mass of $b$ quark is taken equal to $m_{b}=5085 \mathrm{MeV}$. As in the case of charmed $P$-wave $(70,1-)$ baryon, in the case of bottom $P$-wave baryons we have to shift mass from their usual meanings by $245 \mathrm{MeV}(\Delta=1900-1655=245=5085-4840)$.

This shift takes into account the confinement potential and allows baryons to remain below the threshold.

The gluon coupling constants $g_{+}=0.69, g_{-}=0.3$ and cutoff $\Lambda_{u u}=14.5$ for the light quarks are the similar to ones in work [10].

There are only two experimentally known masses of bottom $P$-wave baryons: $\Lambda_{b} \frac{3^{-}}{2}$ $M=5920 \mathrm{MeV}$ and $\Lambda_{b} \frac{1^{-}}{2} \quad M=5912 \mathrm{MeV}$.

We use them to define the parameters for bottom diquarks: $g_{u b, b b}=1.24$, $\Lambda_{u b}=9.1, \Lambda_{b b}=4.82\left(\Lambda_{u b}=\frac{1}{4}\left(\sqrt{\Lambda_{u u}}+\sqrt{\Lambda_{b b}}\right)\right.$ as usual $)$.

\section{Conclusions}

The relativistic three-quark equations of the excited (70,1-) charmed baryons are found in the framework of the dispersion relation technique. The masses of negative parity (70,1-) bottom nonstrange baryons are calculated in the relativistic quark model. We calculated 21 different baryon masses of the (70,1-) bottom baryon multiplet.

Table 1. The $\Lambda_{b}$-hyperon masses of multiplet (70,1-).

\begin{tabular}{|cc|c|c|c|}
\hline Multiplet & Baryon & Mass $(\mathrm{MeV})$ & Mass $(\mathrm{MeV})$ (exp.) \\
\hline$\frac{5}{2}^{-}(8,4)$ & $D_{05}$ & 6027 & - \\
\hline$\frac{3}{2}^{-}(8,4)$ & $D_{03}$ & 5900 & - \\
\hline$\frac{1}{2}^{-}$ & $(8,4)$ & $S_{01}$ & 6159 & - \\
\hline
\end{tabular}




\begin{tabular}{|cc|c|c|c|}
\hline$\frac{3}{2}^{-}(8,2)$ & $D_{03}$ & 5920 & 5920 \\
\hline$\frac{1}{2}^{-}(8,2)$ & $S_{01}$ & 5912 & 5912 \\
\hline$\frac{3}{2}^{-}(1,2)$ & $D_{03}$ & - & - \\
\hline$\frac{1}{2}^{-}(1,2)$ & $S_{01}$ & - & - \\
\hline
\end{tabular}

Table 2. The $\Sigma_{b}$-hyperon masses of multiplet (70,1-).

\begin{tabular}{|cc|c|c|c|}
\hline Multiplet & Baryon & Mass $(\mathrm{MeV})$ & Mass $(\mathrm{MeV})$ (exp.) \\
\hline$\frac{3}{2}^{-}$ & $(10,2)$ & $D_{13}$ & 4057 & - \\
\hline$\frac{1}{2}^{-}(10,2)$ & $S_{11}$ & 5220 & - \\
\hline$\frac{5}{2}^{-}$ & $(8,4)$ & $D_{15}$ & 4649 & - \\
\hline$\frac{3}{2}^{-}$ & $(8,4)$ & $D_{13}$ & 4057 & - \\
\hline$\frac{1}{2}^{-}$ & $(8,4)$ & $S_{11}$ & 5220 & - \\
\hline$\frac{3}{2}^{-}$ & $(8,2)$ & $D_{13}$ & 4161 & - \\
\hline$\frac{1}{2}^{-}$ & $(8,2)$ & $S_{11}$ & 4523 & \\
\hline
\end{tabular}

Table 3. The $\Xi_{b b}$-hyperon masses of multiplet (70,1-).

\begin{tabular}{|c|c|c|c|}
\hline Multiplet & Baryon & Mass $(\mathrm{MeV})$ & Mass $(\mathrm{MeV})(\exp )$. \\
\hline$\frac{3^{-}}{2} \quad(10,2)$ & $D_{13}$ & 9586 & - \\
\hline$\frac{1}{2}^{-} \quad(10,2)$ & $S_{11}$ & 10394 & - \\
\hline$\frac{5^{-}}{2} \quad(8,4)$ & $D_{15}$ & 9483 & - \\
\hline
\end{tabular}




\begin{tabular}{|cc|c|c|c|}
\hline$\frac{3}{2}^{-}$ & $(8,4)$ & $D_{13}$ & 9100 & - \\
\hline$\frac{1}{2}^{-}(8,4)$ & $S_{11}$ & 10016 & - \\
\hline$\frac{3}{2}^{-}(8,2)$ & $D_{13}$ & 9130 & - \\
\hline$\frac{1}{2}^{-}(8,2)$ & $S_{11}$ & 9399 & \\
\hline
\end{tabular}

Table 4. The $\Omega_{b b b}$-hyperon masses of multiplet (70,1-).

\begin{tabular}{|c|c|c|c|}
\hline Multiplet & Baryon & Mass $(\mathrm{MeV})$ & Mass $(\mathrm{MeV})(\exp )$. \\
\hline$\frac{3}{2}^{-} \quad(10,2)$ & $D_{03}$ & 14940 & - \\
\hline$\frac{1}{2}^{-} \quad(10,2)$ & $S_{01}$ & 14984 & - \\
\hline
\end{tabular}

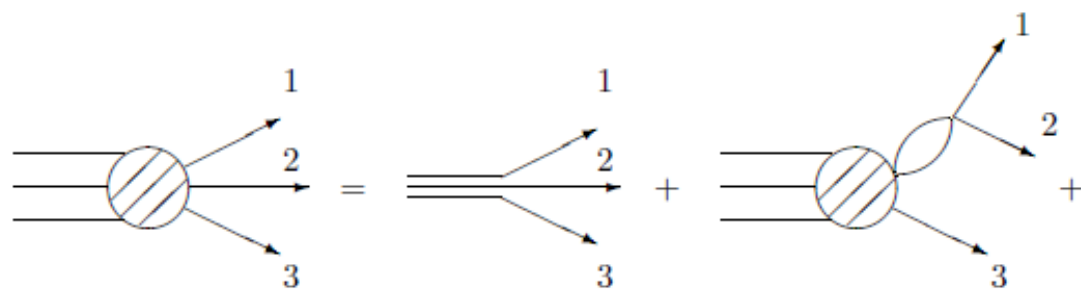

a)

b)

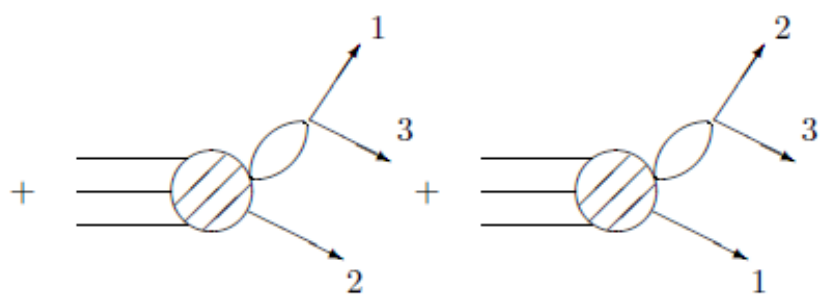

c)

d)

Fig.1. The contribution of diagrams at the last pair of the interacting particles. 

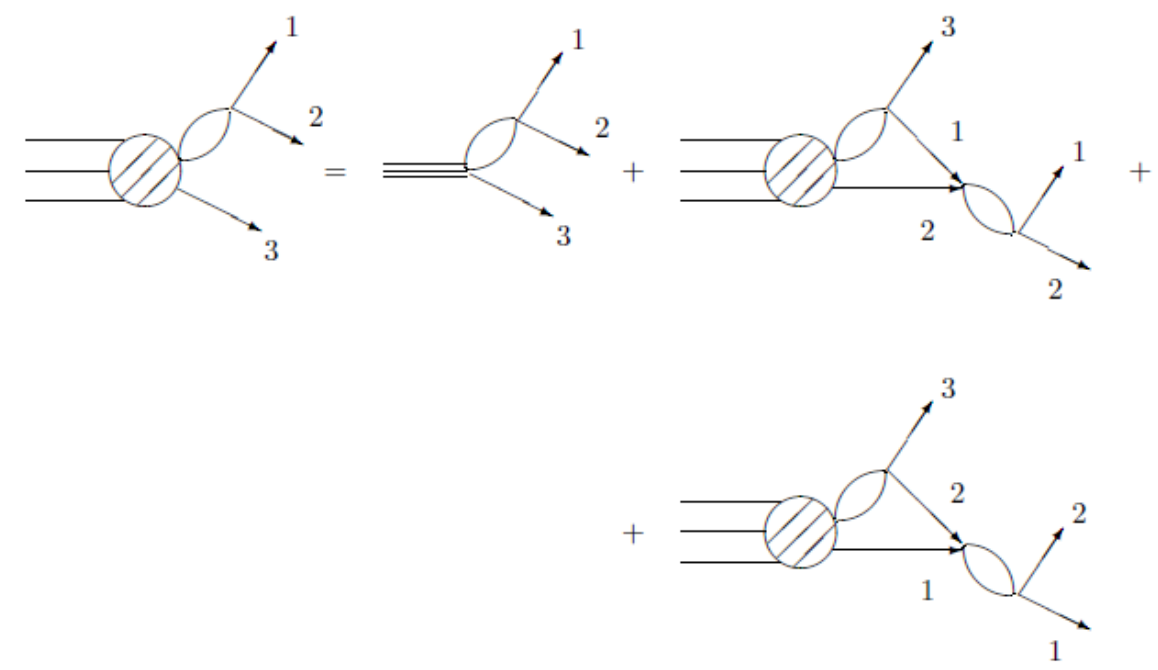

Fig.2. Graphic representation of the equations for the amplitude $A_{1}\left(s, s_{i k}\right)$.
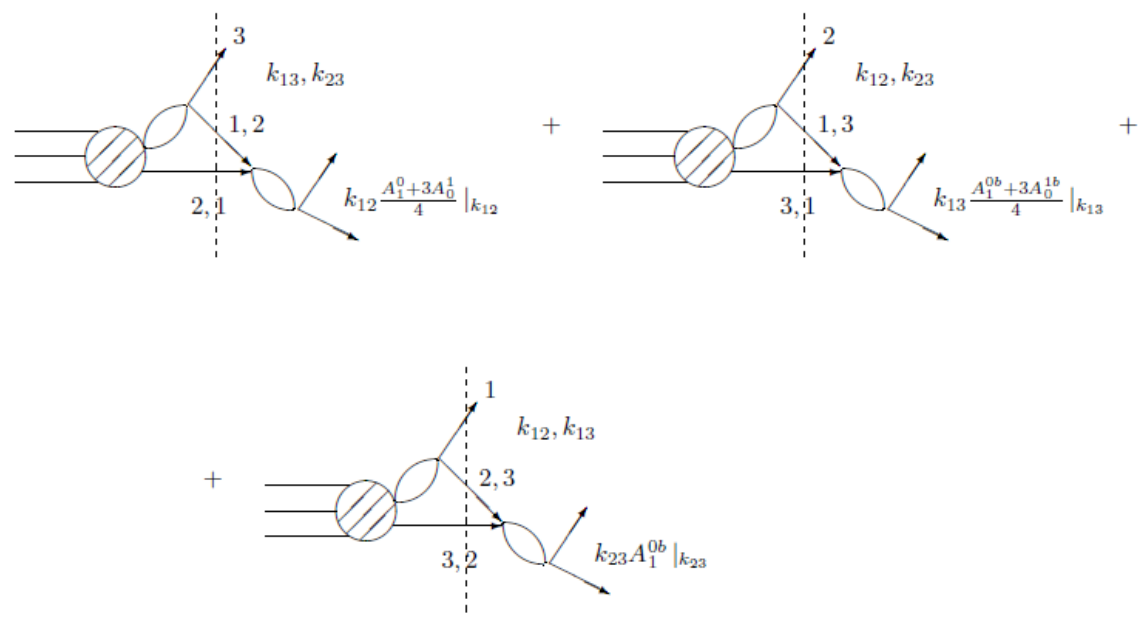

Fig.3. The contribution of the diagrams with the rescattering.

\section{References}

1. G.'t Hooft, Nucl. Phys. B72, 461 (1974) DOI: 10.1016/0550-3213(74)90154-0

2. E. Witten, Nucl. Phys. B160, 57 (1979) DOI: 10.1016/0550-3213(79)90232-3

3. I.J.R. Aitchison, J. Phys. G3 121 (1977) DOI: 10.1088/0305-4616/3/2/006

4. J.J. Brehm, Ann. Phys. (N.Y.) 108454 (1977) DOI: 10.1016/0003-4916(77)90023-9

5. I.J.R. Aitchison, and J.J. Brehm, Phys. Rev. D17 3072 (1978) DOI: $\underline{\text { 10.1103/PhysRevD.17.3072 }}$ 
6. I.J.R. Aitchison, and J.J. Brehm, Phys. Rev. D20 1119, 1131 (1979) DOI: 10.1103/PhysRevD.20.1119, DOI: 10.1103/PhysRevD.20.1131

7. J.J. Brehm, Phys. Rev. D21 718 (1980) DOI: 10.1103/PhysRevD.21.718

8. S.M. Gerasyuta, Z. Phys. C60 683 (1993) DOI: 10.1007/BF01558397

9. S.M. Gerasyuta, and E.E. Matskevich, Yad. Fiz. 701995 (2007) DOI: 10.1134/S1063778807110154

10. S.M. Gerasyuta, and E.E. Matskevich, Int. J. Mod. Phys. E17, 585 (2008) DOI: 10.1142/S0218301308010027

11. G. Chew, and S. Mandelstam, Phys. Rev. 119, 467 (1960) DOI: 10.1103/PhysRev.119.467

12. A.De Rujula, H.Georgi, and S.L.Glashow, Phys. Rev. D12 147 (1975) DOI: 10.1103/PhysRevD.12.147

13. V.V. Anisovich, S.M. Gerasyuta, and A.V. Sarantsev, Int. J. Mod. Phys. A6 625 (1991) DOI: $10.1142 / \mathrm{S} 0217751 \mathrm{X} 9100037 \mathrm{X}$

14. S.M. Gerasyuta, and D.V. Ivanov, Nuovo Cim. A112, 261 (1999) DOI: $\underline{10.1007 / \mathrm{BF} 03035848}$ 